\title{
INTEROPERABILITY ISSUES IN BULGARIA
}

\author{
K. Kirilova* \\ University of National and World Economy, Sofia
}

\begin{abstract}
Activities in public administration are related to the exchange of different in term of purpose and structure documents. If any administrative authority or participant in the system uses its own information system for exchanging electronic documents it would be very difficult this exchange to work properly due to structural differences between documents. Such reasons enforce the use of uniform technology standards between administrations and the providing of opportunities for the exchange of electronic documents through a single environment. Interoperability can be defined as the ability of information systems to process, store and share files and data with each other using uniform technology standards and processes. The paper explains basic challenges for achieving interoperability in Bulgaria and on this basis conclusions are drawn.
\end{abstract}

Key words: E-government, interoperability, public administration.

\section{INTRODUCTION}

Interoperability is a complicated issue for all information systems in state and local government (1). It arises from the need for systems to be able free to exchange data during certain rules with the same sense. There are some major challenges involved in building information systems in public administration. They are related to the need to ensure heterogeneity of processes and ensuring heterogeneity of system implementations. This makes it necessary previously to be determined heterogeneity of structure and presentation of data. Another challenge is to ensure a dynamic business and technology environment and secure and reliable data exchange.

\section{CHARACTERISTICS AND TYPES OF INTEROPERABILITY}

Interoperability can be defined as the ability of information systems to process, store and share files and data with each other using uniform technology standards and processes. Interoperability has various requirements, most of which are defined by ordinances and regulations. Some of them regulate the conditions for integration of national systems with those of the Member - States of the European Union to provide cross-border electronic public services. Interoperability is

* Correspondence to: Katia Kirilova, University of National and World Economy, Sofia 1700, e-mail: katia.kirilova@unwe.bg possible only on the basis of adopted and enforced uniform standards and rules. Administrative authorities are obliged to observe them. They establish the technological and functional parameters that are supported by their information systems to achieve interoperability.

The main types of interoperability are (Figure 1):

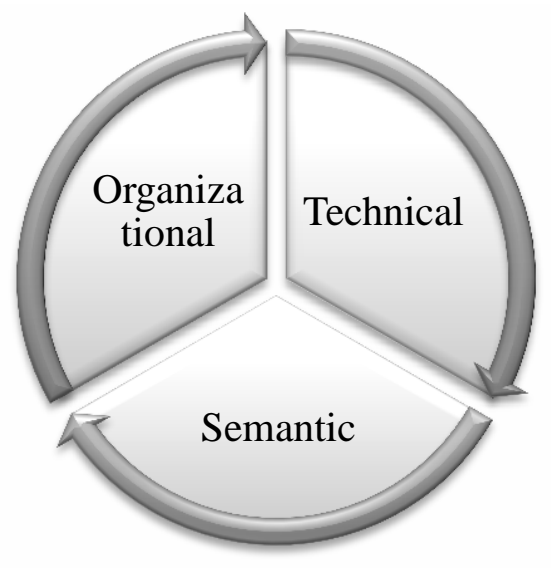

Figure 1. Types of interoperability

- Technical - allowing exchange between application systems in different computers;

- Semantic - providing the same semantic content of the data exchanged;

- Organizational - creating organization management processes for the exchange and processing of data between different organizational structures. 
METHODOLOGICAL ISSUES OF SEMANTIC INTEROPERABILITY
Interoperability of the exchange of electronic documents between administrative bodies is provided through (Figure 2) $(2,3)$ :

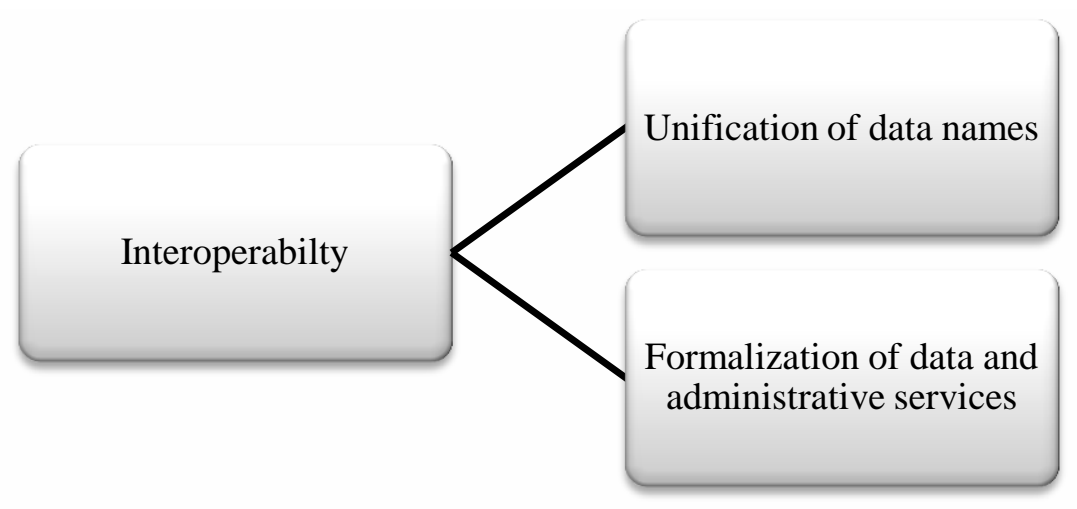

Figure 2 Interoperability

- Unifying the names of the data to be stored in databases or registers;

- Formalization of data and administrative services to ensure technology that enables automated exchanges between administrative authorities and processing of data. The formalized data and formalized description of the electronic administrative services shall be entered in the register of information objects, respectively, in the register of e-services.

The design of the level of realization of semantic interoperability is a complex process. It can be realized by a set of series-connected actions on the design of the individual components (Figure 3):

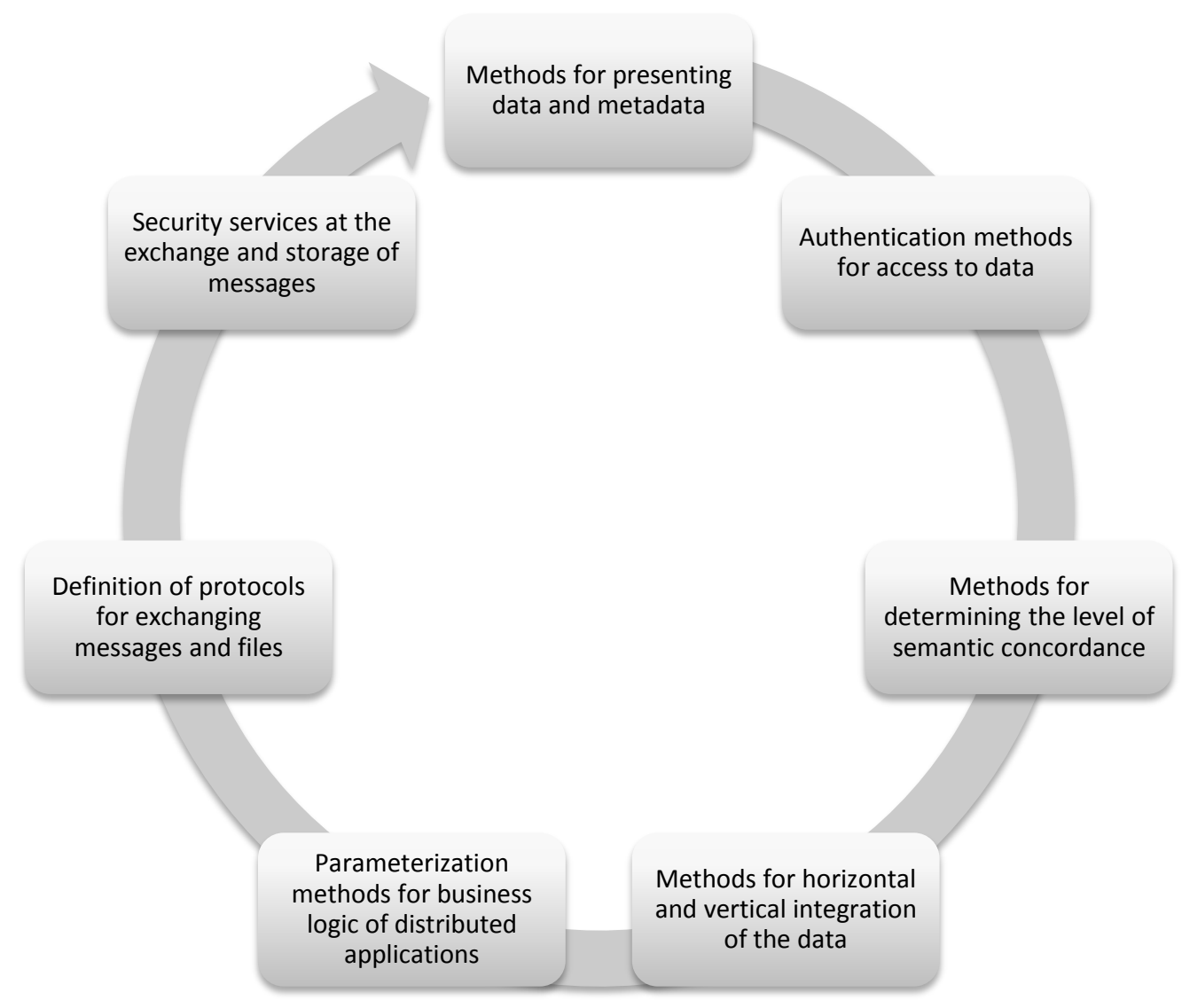

Figure 3. Methodological issues of semantic interoperability 
- Methods for presenting data and metadata;

- Authentication methods for access to data;

- Methods for determining the level of semantic concordance;

- Methods for horizontal and vertical integration of the data;

- Parameterization methods for business logic of distributed applications;

- Definition of protocols for exchanging messages and files;

- Security services at the exchange and storage of messages.

\section{REGISTERS FOR INTEROPERABILITY}

From the perspective of the E-Government Act (4) obligations of the administrative authorities shall apply to persons performing public functions and organizations providing public services in the provision of internal electronic administrative services. Service functions of interoperability are presented through a developed portal of the State Administration for interoperability. This portal presents several basic registries relating to interoperability, as well as their application in the exchange of electronic documents. They are (5):

- Register of standards. Register of standards is kept as a single centralized electronic database managed by the information system containing technical standards and their applicability. It fits the technical standards that must be applied by the administrative authorities for the provision of administrative services and ensure interoperability, information security and automated exchange of information and documents between administrative bodies. Register of standards is a dynamic structure reflecting current status of the standardization process and the possibilities for their application at the current time. Maintaining and updating take place from structure containing expert and executive part by regulated procedures. The main criteria for the selection of the standards are as follows: openness; level of adoption and maintenance; maturity (considering the five levels of efficiency and applicability of the standard); potential; applicability to national circumstances.

- Register of information objects. The register of information objects is conducted as a single centralized electronic database managed by the information system containing a description of all data objects through a particular technical standard. Under data objects we understand a single or multiple data collected, created, stored or processed in exercise of the powers of an administrative body. Keeping, preservation and access to the register of information objects and standard setting is regulated by an ordinance for records of information sites and electronic services by the Council of Ministers. Initial technical description and registration in the register of each data object is made by the Minister of Transport, Information Technologies and Communications. In its work any administrative authority declares for entry standardized descriptions of each information object that collects or creates it;

- Register of electronic services. Register of electronic services is conducted as a single centralized electronic database managed by the information system containing a description by a certain technical standard on all available through a single environment for data exchange electronic administrative services and internal electronic administrative services and in accordance with standardized descriptions of information objects within the register of information objects. For these reasons, any administrative authority declares for registration of electronic services standardized descriptions of all of its provided administrative services and internal electronic administrative services. The relevant competent authority (Ministry of Transport, Information Technology and Communications) examines the compliance of standardized descriptions of standards and descriptions of existing electronic administrative services and internal electronic administrative services. In case of discrepancies, the minister with the administrative authority declared the entry, is capable of removing inconsistencies.

According to the defined interoperability rules all information systems of the administrative authorities should maintain functionality and interfaces for automated submission, respectively service, standardized requests for administrative services electronically. It is important to be noticed that other authors have also explored the possibilities for maintaining various data in a uniform way in order to ease verification of data and complex search for specific information within this data $(6,7)$.

\section{CONCLUSIONS}

The paper dealt with the issues of interoperability in the meaning of connecting 
information systems of state administration. Particular attention is paid to the processes of designing the level of semantic interoperability. They are presented as a sequence of actions in the design and implementation of methods for presenting data and metadata and authentication methods for access to data. The latter are the basis for the development of methods for determining the level of semantic concordance and methods for horizontal and vertical integration of the data. Additional methods are discussed as they are methods for parameterization of business logic of distributed applications, definition of protocols for exchanging messages and files and security services for exchange and storage of messages.

\section{REFERENCES}

1. http://interoperability.egov.bg/

2. The Ordinance on the integrated environment for exchange of electronic documents, http://www.egov.bg/eregpublic/misc/norm/download.rg?_eregId=6
3. Ordinance on the registers of information objects and electronic services, http://www.egov.bg/eregpublic/misc/norm/download.rg?_eregId=1

4. E-Government Act

5. http://www.egov.bg/ereg-public/home.rg

6. Kirilov R., Information problems at verification of public projects, 4th International Conference on Application of Information and Communication Technology and Statistics in Economy and Education (ICAICTSEE 2014), University of national and world economy, Sofia, 2014.

7. Milev P., Technological approaches for researching content of web forums, 4th International Conference on Application of Information and Communication Technology and Statistics in Economy and Education (ICAICTSEE 2014), University of national and world economy, Sofia, 2014. 